\title{
Self-healing ionic gelatin/glycerol hydrogels for strain sensing applications
}

\author{
David Hardman (1)', Thomas George Thuruthel ${ }^{1}$ and Fumiya lida ${ }^{1}$
}

\begin{abstract}
Soft sensing technologies have the potential to revolutionize wearable devices, haptic interfaces and robotic systems. However, there are numerous challenges in the deployment of these devices due to their poor resilience, high energy consumption, and omnidirectional strain responsivity. This work reports the development of a versatile ionic gelatinglycerol hydrogel for soft sensing applications. The resulting sensing device is inexpensive and easy to manufacture, is self-healable at room temperature, can undergo strains of up to $454 \%$, presents stability over long periods of time, and is biocompatible and biodegradable. This material is ideal for strain sensing applications, with a linear correlation coefficient $R^{2}=0.9971$ and a pressure-insensitive conduction mechanism. The experimental results show the applicability of ionic hydrogels for wearable devices and soft robotic technologies for strain, humidity, and temperature sensing while being able to partially self-heal at room temperature.
\end{abstract}

\section{Introduction}

Soft sensing technologies provide a minimally invasive way to measure the internal states of a system and have several applications in health monitoring, human motion detection, human-machine interfaces, and soft robotics $^{1,2}$. Typically, these soft sensors are developed using conductive composites of elastomers ${ }^{3-6}$, conductive liquids $^{7,8}$, and stretchable optical fibers ${ }^{5}$. Hydrogels are an appealing alternative due to their high stretchability, transparency, biocompatibility ${ }^{9,10}$, and ionic conductivity, which can transmit high-frequency electrical signals over long distances. The physical properties of these materials can be easily tuned ${ }^{11-13}$, they are sensorizable with a variety of additives ${ }^{14-17}$, and they demonstrate unique diffusion-based actuation ${ }^{18-20}$. However, their high water content and often organic nature lead to difficulties in ensuring stability and durability over extended time periods ${ }^{10}$. Parasitic impedance layers can cause issues in fabricating an effective interface between the hydrogel and the electrode ${ }^{21}$, causing unwanted noise in the measurement devices. Additionally, as the migration of mobile

\footnotetext{
Correspondence: David Hardman (dsh46@cam.ac.uk)

${ }^{1}$ Department of Engineering, Bio-Inspired Robotics Lab, Cambridge CB2 1PZ, UK
}

ions in the hydrogel network is negligibly impacted by shape changes, the electrical resistivities of these hydrogels remain constant, thereby making them sensitive to changes in both length and area ${ }^{10,22}$. This feature complicates their applicability to strain sensing, as applied stresses in multiple directions cannot be decoupled. For hydrogel-based strain sensors to be truly effective, we need to reduce the vitrification effects that occur due to drying of the hydrogel, improve their robustness to damage, and be able to tune the strain response in desired directions. Additional properties such as biocompatibility, self-healing, low cost, ease of fabrication, and low energy consumption are beneficial.

Additive manufacturing methods have already proved useful in fabricating sensorized hydrogels ${ }^{23,24}$, although specialized/high-cost printers limit the practicality of this method. More straightforward deposition printing methods necessitate extrudable hydrogels ${ }^{25,26}$, which require careful tuning of the material properties and gelation time $^{27}$. Gelatin-based hydrogels are inexpensive, extrudable, and biocompatible due to gelatin's nontoxic nature $^{28-30}$ and can be developed into self-healable materials $^{30-32}$. In this paper, we tune the composition of a gelatin/glycerol/water/citric acid mixture to ensure 
durability, stability, and printability before testing a number of sensorization additives to create composite biocompatible sensors and sensorized actuators, which can be quickly and straightforwardly fabricated.

Upon cooling, aqueous gelatin undergoes a sol-gel transition at $\sim 40{ }^{\circ} \mathrm{C}$, during which its coiled chains transition toward right-handed triple helices ${ }^{31,33}$ to form a soft gel stabilized by hydrogen bonds ${ }^{34}$. This hygroscopic material undergoes adsorption and desorption to match the relative humidity of its surroundings ${ }^{35}$, although over longer periods of time, the hydrogel vitrifies, becoming dry and brittle upon the exclusion of aqueous solvent ${ }^{36}$. The physical crosslinks formed during this gelation are thermoreversible, but additional enzymatic crosslinking can be induced using transglutaminase to facilitate peptide bond formation ${ }^{37,38}$. Although this further crosslinking results in a loss of thermoreversibility ${ }^{31}$, Bigi et al. noted that crosslinked gels have a significantly increased Young's modulus while remaining flexible ${ }^{34}$. The authors also identified a linear relationship between the gel triple helix content and the gelatin bloom index, with higher indices giving stronger gels, higher Young's moduli (3.6 to $12 \mathrm{MPa}$ from Gel-80 to Gel-270), and reduced swelling. To further improve the mechanical properties of the gel and to counter vitrification effects, glycerol may be introduced as a plasticizer ${ }^{15,39}$. Although glycerol is a nonsolvent for gelatin, its three hydroxyl groups form hydrogen bonds with water molecules to increase the bound-water content of the material (Fig. 1). These strong bonds disrupt the formation of ice crystals, and glycerol has been found to be an effective antifreeze additive for hydrogels ${ }^{40}$. Additionally, the presence of these hydroxyl groups was proposed by Pan et al. as an explanation for the self-healing behaviors of a guar gum-glycerol hydro$\mathrm{gel}^{25}$, the thixotropic nature of which makes it a good candidate for extrusion-based hydrogel printing.

When used in conjunction with gelatin hydrogels, glycerol does not affect the gelation mechanism ${ }^{36}$ but improves elasticity, flexibility, and moisture sensitivity by reducing interactions between adjacent gelatin chains ${ }^{41}$. Sanwlani et al. proposed a "hydration sheath" mechanism by which glycerol acts as a water structure enhancer to improve gel strength ${ }^{33}$, finding a linear relationship between glycerol concentration and gel strength in high solvent mixes. The relative proportions of gelatin (Ge), glycerol (Gl), and water in the mixture can be used to tune the mechanical properties of the hydrogel ${ }^{42}$. Shintake et al. tested a series of samples using Ge: $\mathrm{Gl}: \mathrm{H}_{2} \mathrm{O}$ ratios of 1:1:8 and 1:2:8 with a $48 \mathrm{~h}$ drying time but reduced the water content to $1: 2: 3$ when casting pneumatic actuators $^{39}$. Nagai et al. demonstrated the biodegradability of 2:1:8 films in soil over a period of days ${ }^{43}$. Hughes et al. utilized a higher glycerol content (1:6:5) to create a material that biodegrades in warm water ${ }^{44}$. Ratios of
1:1.5:8 are suitable for extrusion-based printing when the enzyme transglutaminase is used to catalyze the gelation process ${ }^{45}$. However, the accompanying changes in viscosity lead to low resolution of the printed structures. Baumgartner et al. ${ }^{46}$ found that the acidic environment created by a small amount of citric acid-a food-safe ingredient-prevents bacterial growth without mechanical degradation in a hydrogel of composition 1:2:2:1.75:0.25 Ge:Gl: $\mathrm{H}_{2} \mathrm{O}: G S: C A$, where CA represents citric acid and GS represents glucose syrup.

In this work, a 1:1.5:2.5:0.2 Ge:Gl: $\mathrm{H}_{2} \mathrm{O}: \mathrm{CA}$ composition is selected to optimize the hydrogel printability and stability. Although the pure mixture shows a strong strain response, the baseline resistance of the hydrogel must be lowered to fabricate efficient and soft strain sensors. Four additives are tested that not only reduce the baseline resistance but also maintain the material's mechanical properties, biodegradability, and relative sensitivity. Sodium chloride is found to give excellent results, creating a sensorized hydrogel capable of $454 \%$ strain, room temperature self-healing, and linear correlation coefficient $R^{2}=0.9971$ over $300 \%$ strain (Fig. 1). Our hydrogel remains functional over a period of multiple months without drying out, is composed entirely of widely available, food-safe materials-including the sensorization additive-and exhibits extremely linear strain responsivity over large deformations. In addition to presenting the material composition and properties, we utilize a low-cost fabrication technique consisting of a modified desktop 3D printer, which allows the development of custom strain sensors that can be printed directly onto flexible substrates.

To justify the use of the hydrogel for rapid strain sensor prototyping, its behaviors directly after printing are characterized as an environmental equilibrium is approached. To exploit quick fabrication for customizable sensors or sensor morphology optimizations, it is necessary to understand how the sensor behaves within this window; commonly, the material is instead left to reach equilibrium before any measurements are taken ${ }^{38,39}$.

A straightforward dual-nozzle 3D printing setup is introduced, an extension of that proposed in the authors' previous work $^{45}$, which can be used to combine pure and sensorized hydrogels into composite structures. The introduction of a temperature-controlled printing mechanism eliminates the need for chemical crosslinking, ensuring consistent material properties. Experimentally, the mix of 1:1.5:2.5:0.2 Ge:Gl: $\mathrm{H}_{2} \mathrm{O}: \mathrm{CA}$ is found to have a coalescence boundary near $45^{\circ} \mathrm{C}$ and to undergo gelatin's thermoreversible sol-gel transition within $90 \mathrm{~s}$ of printing at room temperature. The relatively low water content allows prints to reach equilibrium more quickly, leading to less temporal variability in the material properties. Coupled with the addition of citric acid monohydrate, the 


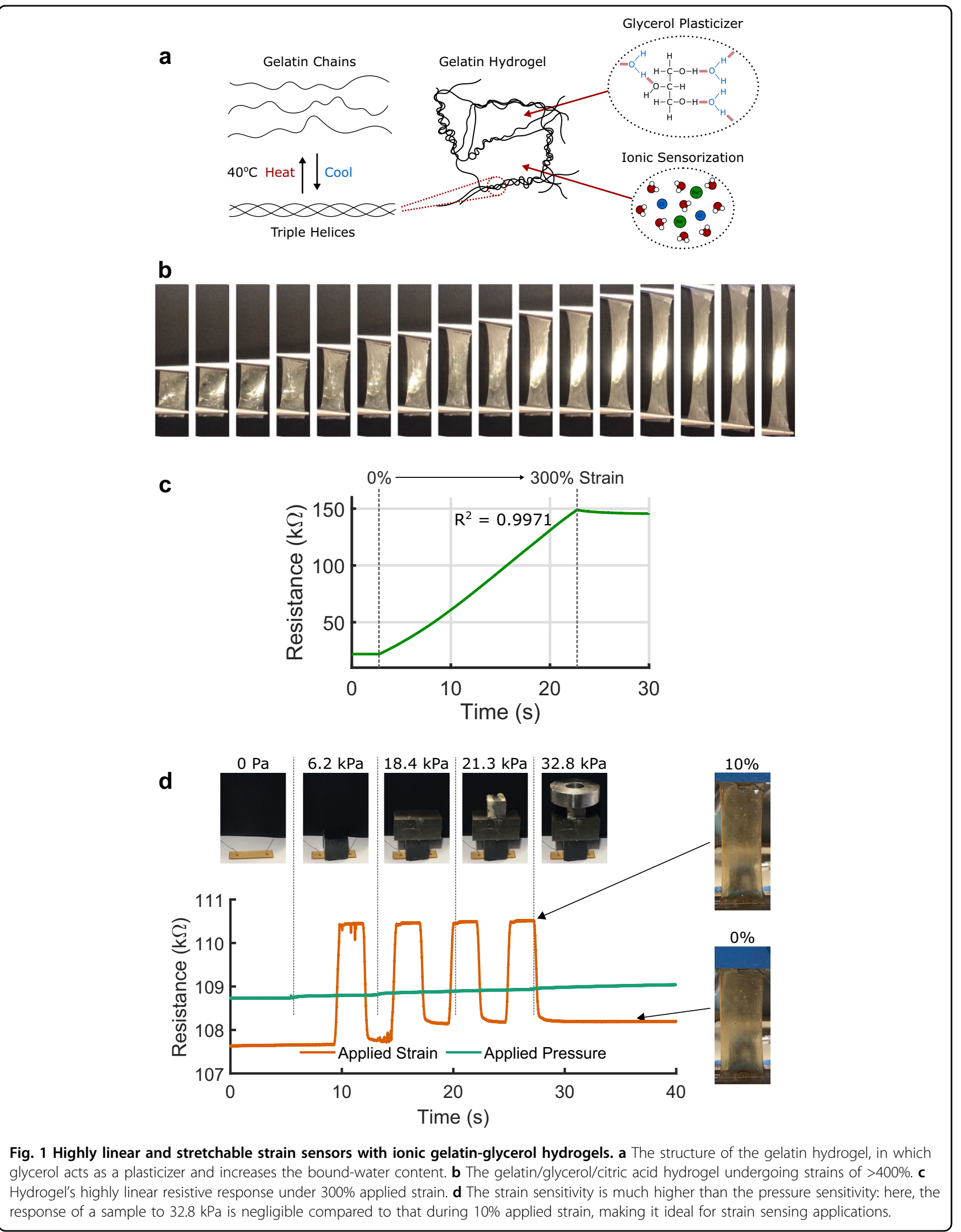


printed samples are found not to degrade over timescales of multiple months. This material stability, coupled with the straightforward and low-cost fabrication method, has major application in the automated design and rapid prototyping of sensorized soft robots. We show that the environmental sensitivity of the electrical properties of the hydrogel can be used to measure these factors, and even compensate for these effects using multiple sensors. Finally, two robotic applications of the sensorized hydrogels are demonstrated: a 3D-printed sensorized wearable glove and a self-healing soft pneumatic actuator with embedded sensing, which remains stable and functional over multiple months.

\section{Results}

\section{Choosing a sensorization additive}

The resulting resistances of the four sensorization additives are compared in Fig. 2c. When evaluated by the ability to decrease the hydrogel's baseline resistance, the ionic additive $\mathrm{NaCl}$ outperforms the other additives over both timescales. Carbon conductive paint (CCP), which has a high water content, has little effect on the resistance at this ratio. Previous studies have demonstrated the ability of CCP to better enhance the hydrogel conductivity at higher ratios ${ }^{45}$, although this comes at the cost of material vitrification in low-humidity environments. Similarly, higher concentrations of $\mathrm{NaCl}$, milled carbon fiber (CF), and graphite powder (GP) are all empirically found to noticeably diminish the material's mechanical properties and are not explored in depth.

At the same concentrations, Table 1 presents the resistive gauge factor (GF) values measured during $e=$ $30 \%$ applied strain (chosen to ensure that all responses remain in an approximately linear response range-limited by graphite powder), where

$$
\mathrm{GF}=\frac{(\underline{\Delta} R)}{R} / e .
$$

The highest gauge factor, indicating the greatest relative response, is that of the pure $\mathrm{Ge} / \mathrm{Gl} / \mathrm{H}_{2} \mathrm{O} / \mathrm{CA}$ hydrogel. Additives are therefore not required to provide a strain sensing mechanism but rather to maintain the sensitivity of the pure mixture, while lowering its baseline resistance to improve the energy efficiency of the sensor network. At the 1:0.02 mass ratio, $\mathrm{NaCl}$ does so most successfully and appears to be the best choice of conductivity-enhancing additive for our purposes.

The samples containing the most water-pure and $\mathrm{CCP}$ mixtures demonstrate the greatest relative resistance increase over the 5-day period. This result suggests that the primary mechanism for increasing resistance is the desorption of water from the samples as they approach equilibrium with the environment. To test this hypothesis,
Fig. $2 \mathrm{~d}$ tracks the relative masses of four equally sized samples. These are created from the same gelatin batch, extruded simultaneously, and weighed over a 7-day period in the laboratory. The pure composition is compared to the mixture with added salt, carbon conductive paint, and without citric acid monohydrate. As expected, the relative mass of the carbon conductive paint sample decreases dramatically, reaching $39 \%$ of its initial value $\sim 40 \mathrm{~h}$ after extrusion. In all samples, small fluctuations are seen around the equilibrium state as the environmental humidity is varied. Although the salted sample loses more water than the pure sample, it demonstrates a much lower increase in relative resistance, suggesting that the ionic conductivity mechanism is less affected by desorption than the pure mixture. Removing citric acid from the mixture has a large effect on the equilibrium mass since the monohydrate form used acts to increase the hydrogel's bound water content, with the hydroxyl and three carboxyl groups providing a number of additional sites for hydrogen bonding. Because we desire our printed sensors to behave as uniformly as possible with time, the $\mathrm{Ge} / \mathrm{Gl} /$ $\mathrm{H}_{2} \mathrm{O} / \mathrm{CA} / \mathrm{NaCl}$ composition is the best choice for printing.

\section{Mechanical sensitivity}

Having chosen $\mathrm{NaCl}$ as an additive, we next examine its effect on the properties of the hydrogel under mechanical loading, with changes in humidity, and over multiple timescales. The alternating current (AC) resistive response of a printed sample containing $1 / 50 \mathrm{NaCl}$ to $300 \%$ strain, applied at a uniform rate of $5 \mathrm{~mm} / \mathrm{s}$, is shown in Fig. 1c. The linearity in response over a large applied strain is very high, with an $R^{2}$ value of 0.9971 . This result is attributed to the material's insensitivity to pressure, which implies an insensitivity to area changes caused by deformation.

Figure 2e, f illustrate the impressive mechanical properties of the pure composition after 7 days, which behaves linearly until failures at $>400 \%$ and $>500 \mathrm{kPa}$. Figure $2 \mathrm{~d}$ desorption behaviors have a clear effect on the strength of the samples: the print given six additional days to reach environmental equilibrium achieves a failure stress $37 x$ higher than that after just 1 day. For the samples printed and measured at high humidity, the addition of salt appears to have little effect on the mechanical behavior until the final moments before fracture (Since the $\mathrm{NaCl}$ sample may have undergone minor slippage in the Instron vice, the slightly lower but more reliable strain value of $454 \%$ is used in Table 2 presentation of sensor properties.). The closely matching responses over $400 \%$ strain show excellent promise for the integration of conductive and insulating layers into 3D structures, while minimizing interfacial failures due to stress concentration. The figure also illustrates the effect of humidity on the mechanical 
a

mass ratio
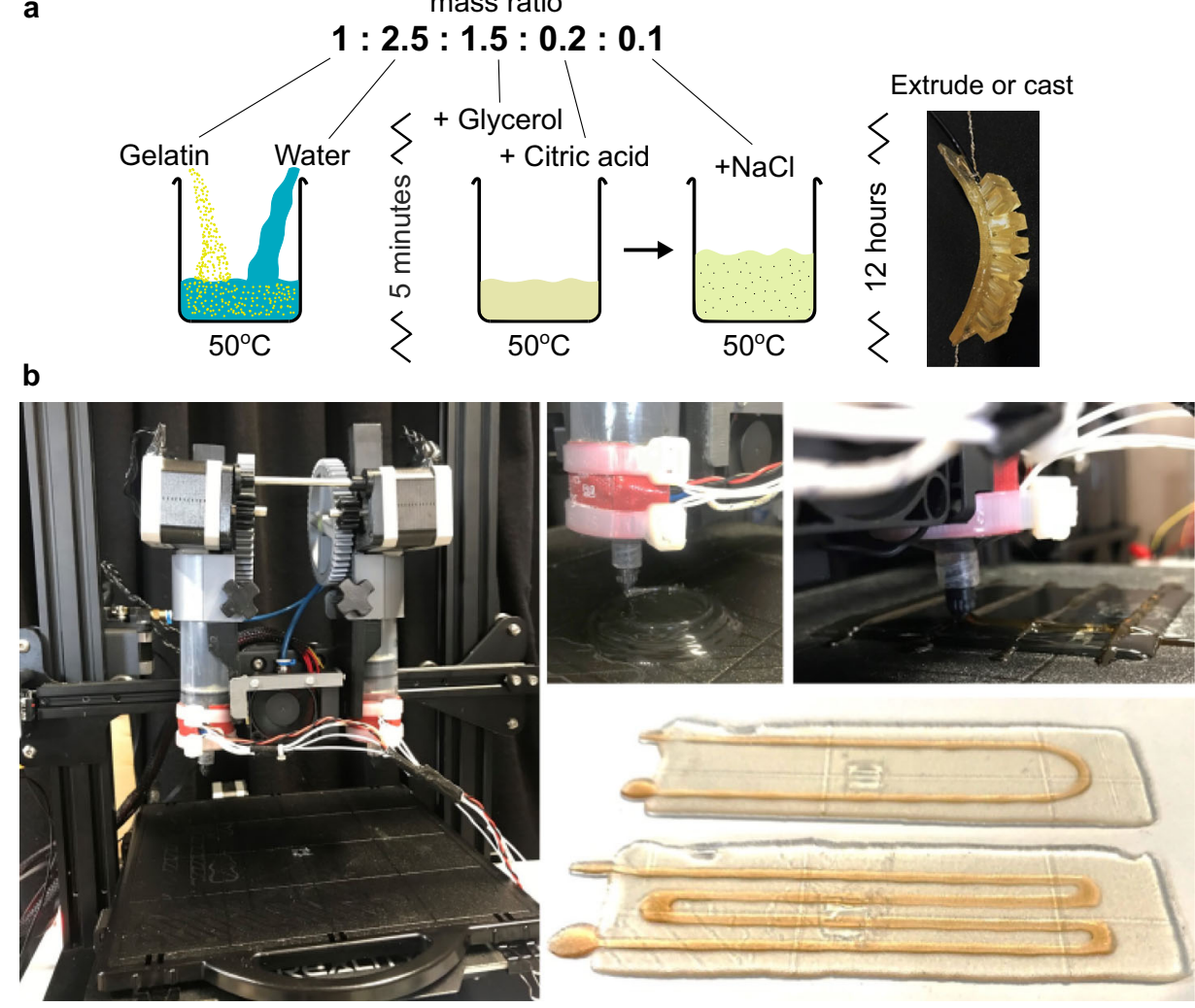

c

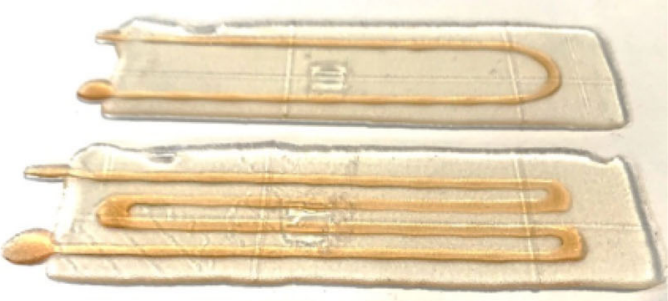

d
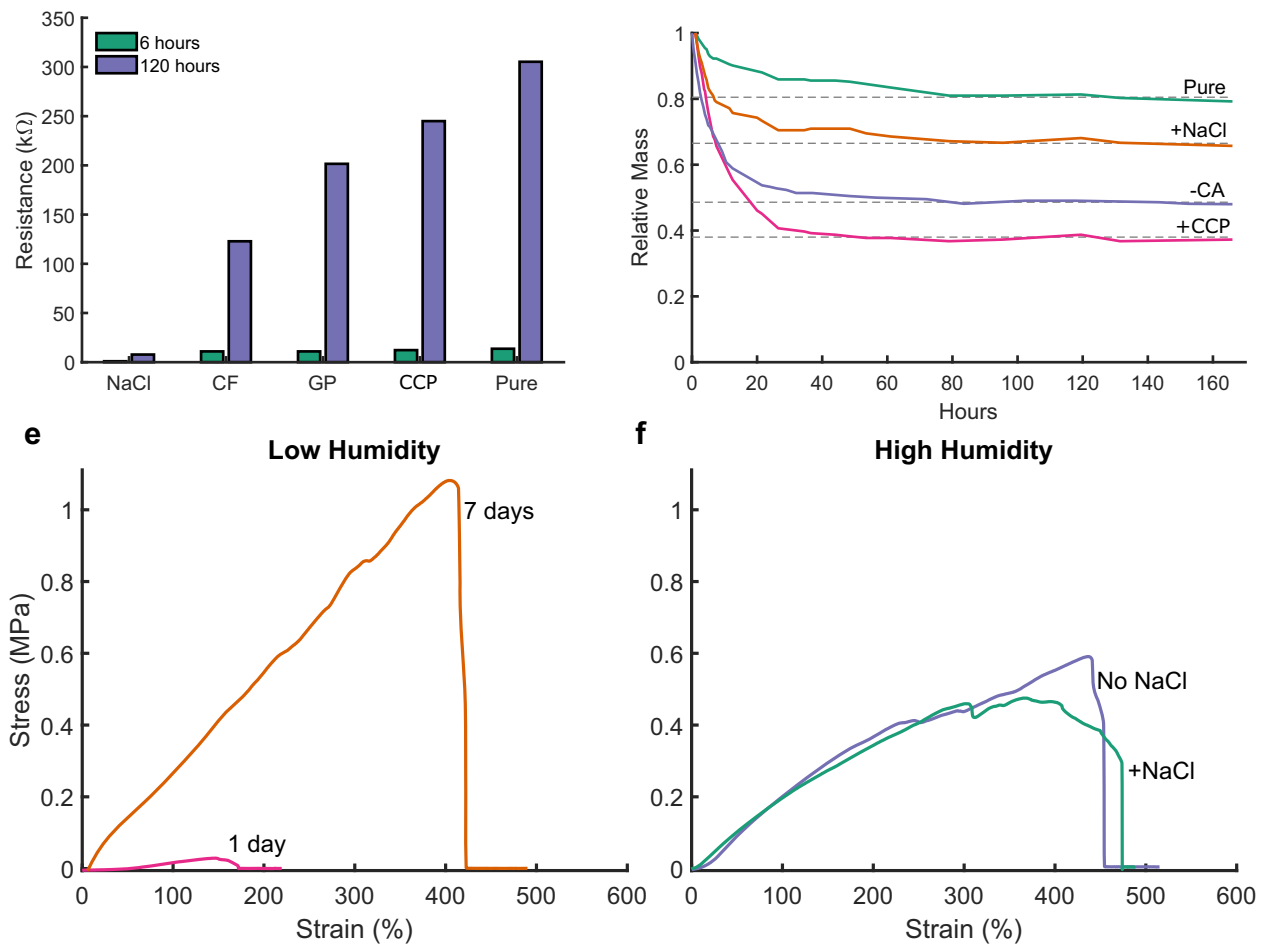

Fig. 2 Fabrication and material selection. a Simple fabrication process of the hydrogel in a water bath at $50^{\circ} \mathrm{C}$. $\mathbf{b}$ Dual-nozzle $3 \mathrm{D}$ printing setup showing printing of a multilayer pyramid and three custom strain sensor morphologies. $\mathbf{c}$ The effect of the four sensorization additives on the hydrogel's baseline resistance. $\mathbf{d}$ Various compositions reaching environmental equilibrium in the hours immediately following printing. $\mathbf{e}, \mathbf{f} L$ Lowhumidity and high-humidity stress-strain responses of the hydrogel over a 1 week time period. e Tests the Ge/Gl/ $\mathrm{H}_{2} \mathrm{O} / \mathrm{CA}$ composition, while $\mathbf{f}$ tests the $1 / 50 \mathrm{NaCl}$ mix after 7 days. 
properties of the hydrogel, which is explored further in Fig. 3c.

Figure 3a tracks the electrical properties of the material over a 6-day period at $1 \mathrm{kHz}$ to various mechanical stimuli. A low pressure sensitivity and high strain sensitivity are desirable for a linear strain sensor, as discussed in the "Introduction" section. The relative responses are remarkably consistent $(\mathrm{GF} \approx 1.5)$ throughout both composition and temporal changes, considering the large changes in baseline resistance during the period. $\mathrm{NaCl}$ can therefore be used to adjust the baseline resistance of a sample while hardly affecting its sensitivity, enabling the fabrication of composite sensor networks: even with different salt contents, a constant gauge factor results in a sensor's resistance always changing by the same proportion to a given strain, such that the baseline resistances of individual components in the network can be tuned to suitable values. The consistency in response is also incredibly beneficial in making meaningful measurements over resistive changes due to environmental changes: only a single measurement is required to recalibrate the sensor when it encounters any environmental fluctuations, from which the same proportional changes are expected. The reduction in resistance to flexural motion is advantageous, as it can provide a simple method to decouple pure bending and strain.

\section{Environmental sensitivity}

The electrical properties of the hydrogel are strongly dependent on temperature and humidity, as observed from our experiments. Although this dependency is not ideal for strain sensing applications, it allows us to develop sensor systems that can estimate multiple physical properties using the same sensing principle. To demonstrate this, we use three gelatin-glycerol hydrogels with $\mathrm{NaCl}$ added at $0,1 / 50$, and $1 / 100$ by weight. The resistances of

Table 1 Measured gauge factors at $10 \mathrm{kHz}$ and $30 \%$ applied strain for different additives.

\begin{tabular}{lllll}
\hline $\mathrm{NaCl}$ & CF & GP & CCP & Pure \\
\hline 1.49 & 1.04 & 1.33 & 1.40 & 1.62 \\
\hline
\end{tabular}

these samples are measured over a 19 day period while sampling the relative atmospheric humidity and temperature. After a $70 \mathrm{~h}$ near-linear initial period in which the hydrogel reaches equilibrium with the environment, a significant increase in relative humidity corresponds to a subsequent decrease in resistance and vice versa. This equilibrium can be sustained for long periods of time: Fig. 3 f shows the material behaviors and responses of a sensorized hydrogel sample 6 months after extrusion. Not only is the hydrogel's flexibility maintained, but a $100 \%$ strain test demonstrates a gauge factor of 1.7 , similar to that of the original tests.

Since each of the samples produces a unique response to the environmental conditions, it becomes easier to decouple and model their dependencies ${ }^{47}$. Figure $3 \mathrm{~b}$ shows how the sensor data can be used along with a learning-based approach to estimate environmental variables and estimate the passive response of other sensors to the environment. The left side of Fig. 3b shows the predictions of a feedforward network that is trained to simultaneously estimate the temperature and relative humidity of the environment from the three previous sensor datasets. Similarly, the environmental effects on one sensor can be removed completely using information from the other sensors. On the top-right side of Fig. 3b, we show how the sensor data from the first two sensors can be used to predict the response of the third sensor. This prediction can then be used to completely remove the effect of environmental factors from the sensors and reliably use it as a strain sensor. The regression fit on a test set is shown for both learned models to verify that the trained model does not overfit the data on the bottomright side of Fig. $3 \mathrm{~b}$.

In addition to the stable strain response, conductivity, strong mechanical response, and printability of the Ge/Gl/ $\mathrm{H}_{2} \mathrm{O} / \mathrm{CA} / \mathrm{NaCl}$ hydrogel, Fig. 3c, d demonstrate its room temperature self-healing capability. In Fig. 3c, a batch of identical rectangular samples are cut into halves using a scalpel at various hours after their extrusion. The halves are placed back into contact at room temperature, and the strain is tested $48 \mathrm{~h}$ later. Tests are also carried out on rehydrated and thermally healed samples over the same period (see "Mechanical/Electrical characterization" section).

Table 2 Key characteristics and material properties of the sensorized hydrogel presented in this work.

\begin{tabular}{llllllll}
\hline & $\begin{array}{l}\text { Sensor } \\
\text { type }\end{array}$ & $\begin{array}{l}\text { Gauge } \\
\text { factor }\end{array}$ & $\begin{array}{l}\text { Linearity } \\
\left(\boldsymbol{R}^{2} \text { value }\right)\end{array}$ & $\begin{array}{l}\text { Maximum } \\
\text { strain }\end{array}$ & $\begin{array}{l}\text { 3D } \\
\text { printability }\end{array}$ & $\begin{array}{l}\text { Material } \\
\text { toxicity }\end{array}$ & $\begin{array}{l}\text { Other } \\
\text { characteristics }\end{array}$ \\
\hline $\begin{array}{l}\mathrm{Ge} / \mathrm{Gl} / \mathrm{H}_{2} \mathrm{O} / \mathrm{CA}+ \\
\mathrm{NaCl}\end{array}$ & $\begin{array}{l}\text { Strain } \\
\text { (Resistive) }\end{array}$ & 1.49 & 0.9971 & $454 \%$ & Extrusion-based & $\begin{array}{l}\text { Biocompatible } \\
\text { food safe } \\
\text { components }\end{array}$ \\
\hline
\end{tabular}



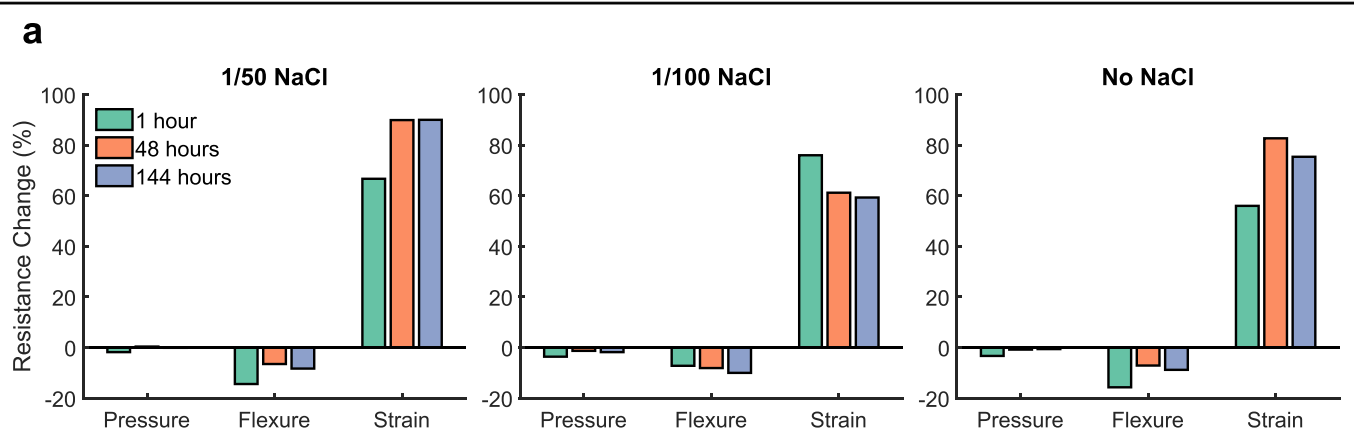

b
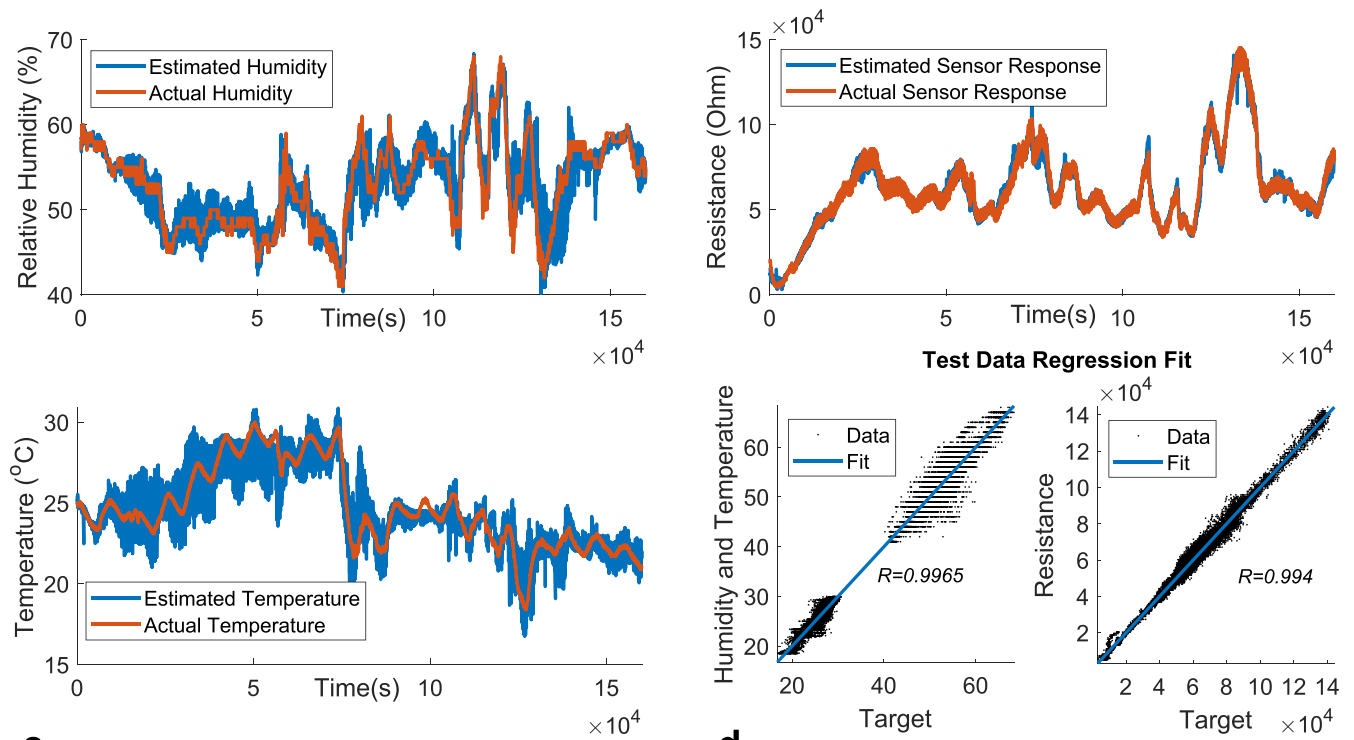

C
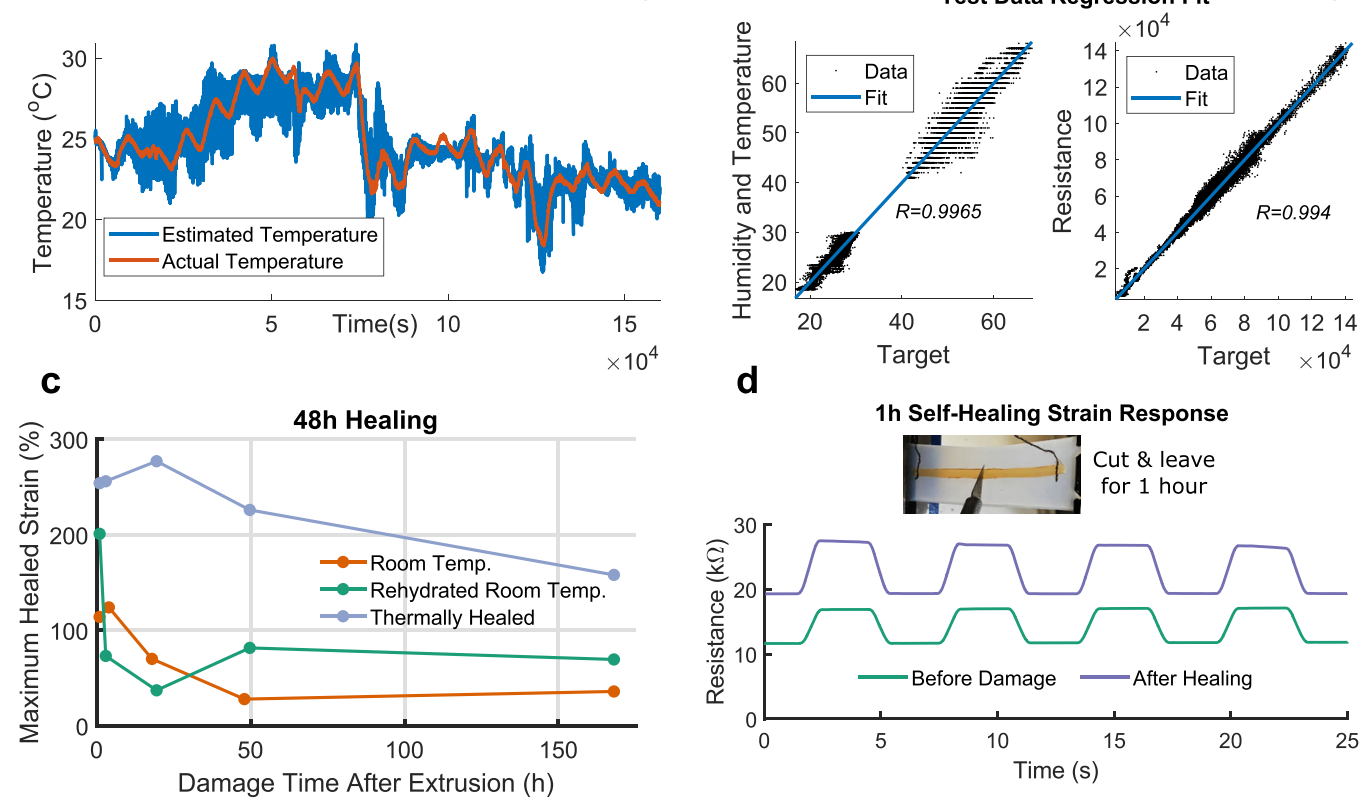

d
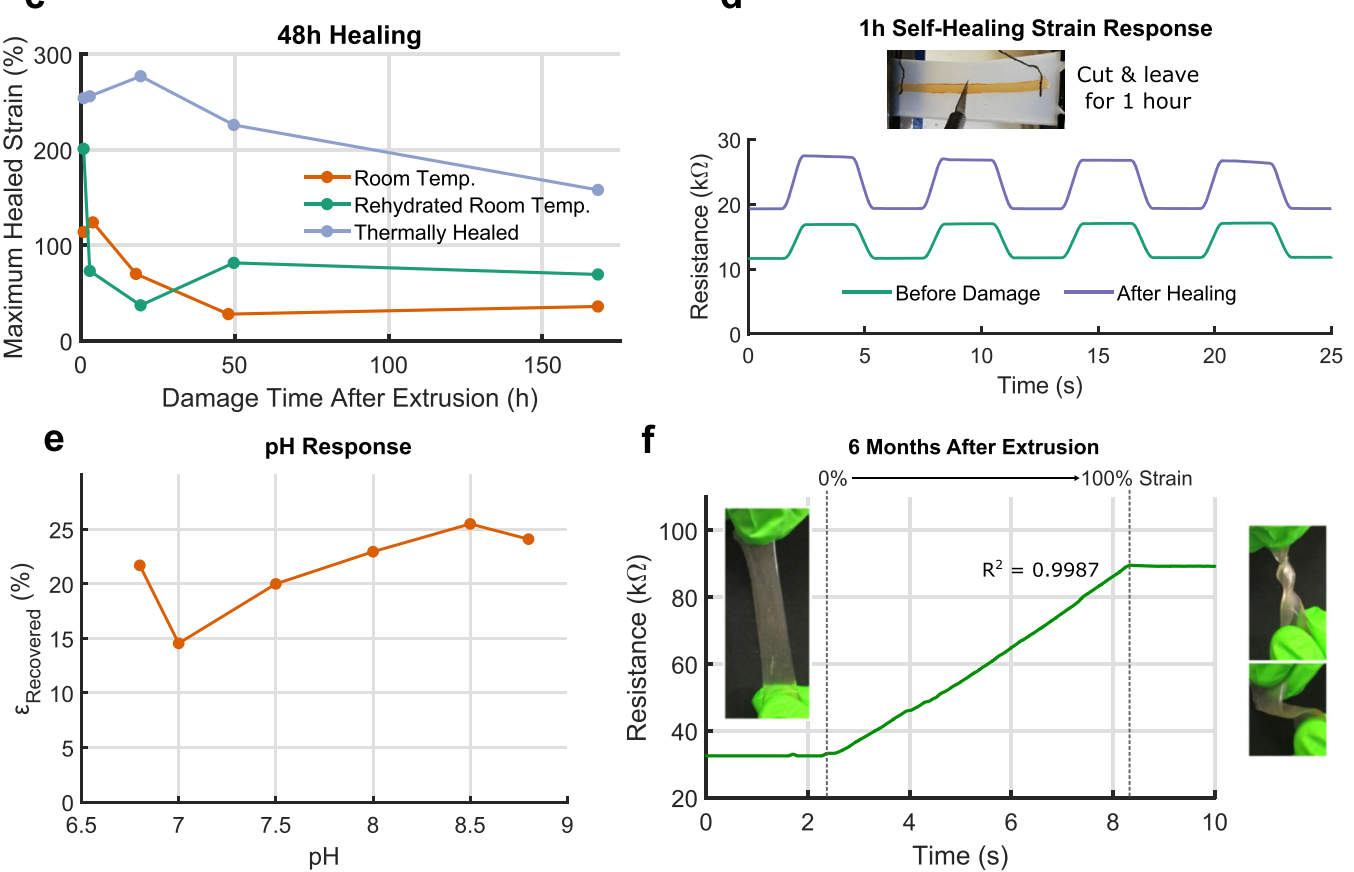

Fig. 3 (See legend on next page.) 
(see figure on previous page)

Fig. 3 Desirable properties and responses of the sensorized hydrogel. a Pressure, flexure, and strain sensitivities of the gelatin mixture at $1 \mathrm{kHz}$, both with and without added salt. $\mathbf{b}$ Hydrogel sensors are sensitive to environmental conditions. This can be used to estimate environmental factors and can be compensated using multiple sensors. c Self-healing: the recovered strains when a sample is cut in half and left at room temperature for $48 \mathrm{~h}$, with no changes, with rehydration, and with thermal healing. $\mathbf{d}$ The recovery of a damaged strain sensor to a trapezoidal strain response in $1 \mathrm{~h}$ at room temperature. e Recovered strain $24 \mathrm{~h}$ after subjection to different $\mathrm{pH}$ values of buffer solution. $\mathbf{f}$ Material stability and sensitivity 6 months after extrusion.

The room temperature self-healing effects are found to be largest when a cut is made during the desorption period (Fig. 2e): upon testing, the samples can undergo up to $124 \%$ applied strain before the onset of fracture at the cut site. When the same test is performed more than 2 days after printing, only $28 \%$ strain is achieved, suggesting that the presence of free water is vital to the selfhealing mechanism; a strong environmental dependence suggests a reliance upon noncovalent interactions ${ }^{48}$. Indeed, after 2 days, the rehydrated samples (given an excess of free water $48 \mathrm{~h}$ before testing) recover higher strains $(\sim 80 \%)$ than the room temperature samples, which have reached equilibrium with the environment. A strain of $80 \%$ allows for a significant range of motion or pneumatic inflation in robotic applications up to a week after extrusion but is still significantly lower than the strain-tofailure of the undamaged material. Further tests are necessary to deduce the reliability of rehydration as a healing "catalyst", such as whether the environmental temperature and humidity significantly affect these results. Due to the thermoreversibility and low sol-gel transition temperature of the hydrogel, thermal healing is capable of recovering the greatest strain $(>150 \%)$ after 1 week, providing a mechanism for more substantial healing after the initial desorption period. Unlike room temperature tests, this method of healing requires an external stimulus (heat), relying on a different mechanism for recovering damage.

The hydrogel's $\mathrm{Ge} / \mathrm{Gl} / \mathrm{H}_{2} \mathrm{O} / \mathrm{CA}$ composition results in a large number of sites for hydrogen bonding, providing a feasible healing mechanism in room temperature samples $^{49,50}$ or part of a multistep mechanism: Xu et al. and Wang et al. $^{51,52}$ previously facilitated self-healing in gelatin hydrogels by introducing additional hydrogen bond donors/receptors with ureidopyrimidinone and tannic acid, respectively. The use of $\mathrm{NaCl}$ and citric acid monohydrate may introduce a further mechanism: $\mathrm{Li}$ et al. identified the high aggregation strength of noncoordinating metal ions and carboxylate anions in selfhealing polymers ${ }^{53}$. In addition, citric acid lowers the hydrogel $\mathrm{pH}$ below the gelatin isoelectric point, such that electrostatic interactions could contribute to the mechanism ${ }^{54}$. Further investigations, including spectroscopic analyses across the healed interface, are necessary to deduce the specific bonding behind the observed behaviors and could provide information to help improve the recovered mechanical properties. A preliminary investigation of the effect of increasing the $\mathrm{pH}$ on any electrostatic healing mechanism (see "Mechanical/Electrical characterization" section) is shown in Fig. 3e. pH values close to 8.5 are found to result in higher recovered strains, although these values are lower than the healed strains tested in Fig. 3c due to the shorter time period between buffer solution subjection and testing. Repeating the testing over multiple timescales is a sensible route for further investigations. At the same concentrations and ranges, these $\mathrm{pH}$ values were not found to significantly impact the sensitivities or baseline resistance of the hydrogel.

Although the samples of Fig. 3c, d fracture at the site of the initial cut, regaining strains of $>100 \%$ at room temperature can still benefit a wide range of scenarios. Figure 3e demonstrates how a soft sensory channel can be repaired in as little as one hour during the strong initial self-healing period. Here, a single line of hydrogel (colored using dye) is extruded onto a silicone substrate, and a $22 \%$ trapezoidal strain profile (typically sufficient for wearable sensors) is applied while resistance is recorded. Figure 3e marks the location at which the sensor is cut with a scalpel. After $1 \mathrm{~h}$, the strain response is rerecorded, regaining full functionality with no increase in baseline resistance beyond that expected due to desorption. Such a quick recovery of sensory response upon damage is necessary for time-limited applications in high-risk situations, such as hostile environment recovery tasks. By coupling the self-healing sensor with a self-healing insulating substrate, restoration of high functionality can be ensured with minimal interfacial/damage-zone stresses.

\section{Applications}

Figure 4 demonstrates two promising applications for $\mathrm{NaCl}$ gelatin sensors: a customizable sensor morphology that can be printed directly onto a wearable glove for human joint tracking and a self-healing sensorized pneumatic actuator that remains stable and functional over multiple months. Figure 4a demonstrates the quickto-fabricate glove morphology using a 3D printing setup to print a sensorized flexible network onto a disposable glove. Due to the excellent adhesive properties of the 
a

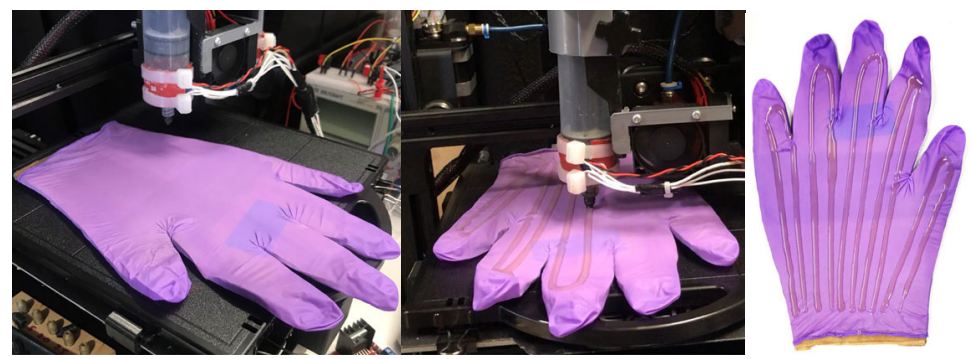

b
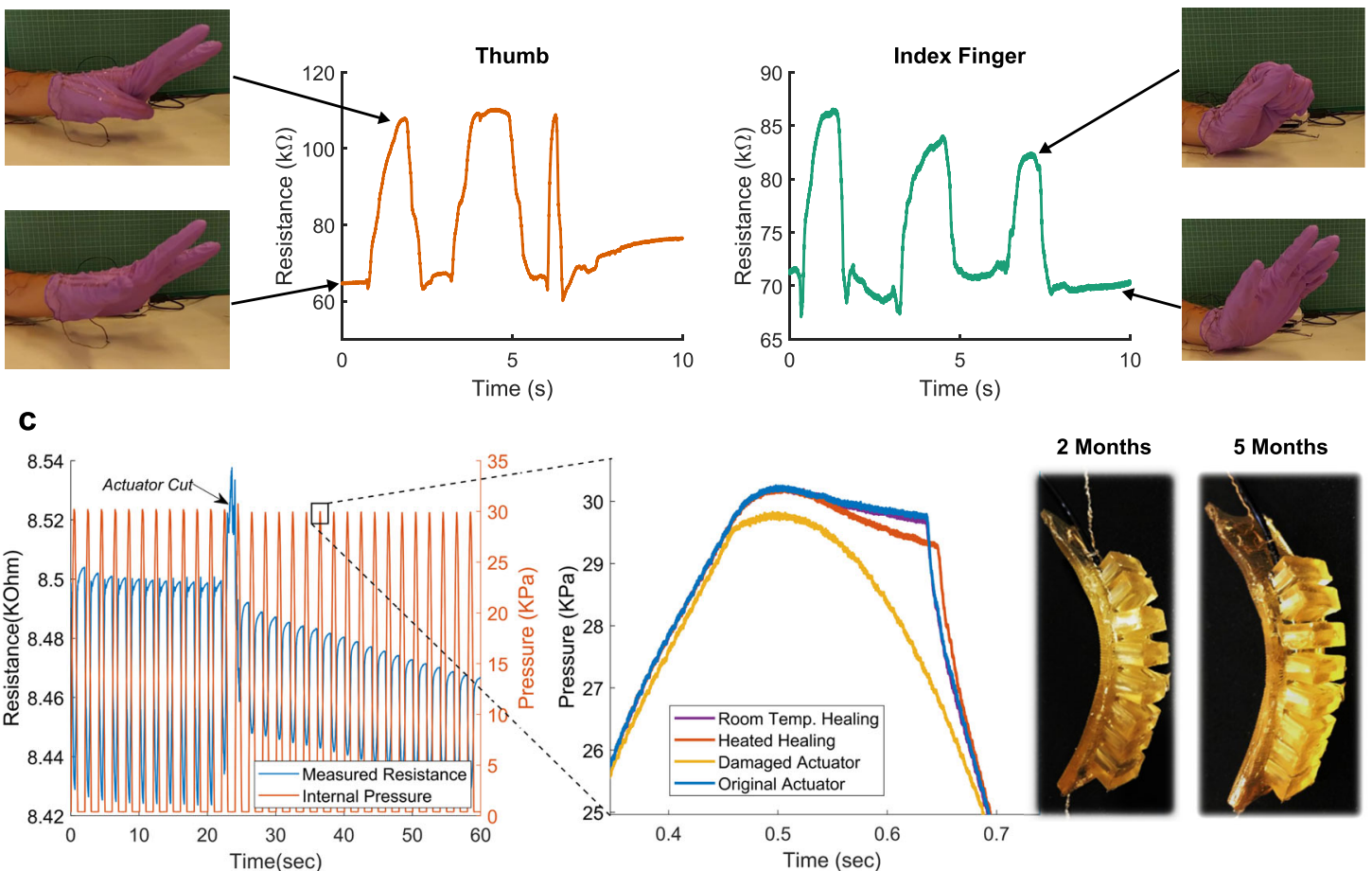

Fig. 4 Wearable and robotic applications of the sensorized hydrogel. a A custom sensor morphology is printed directly onto the surface of a nitrile glove. $\mathbf{b}$ All five fingers of the glove return separate strain responses, enabling proprioceptive data to be inferred. c A cast PneuNet actuator demonstrates the healing of a scalpel cut after $30 \mathrm{~min}$ at room temperature and after a small amount of manual reheating. The actuator is pictured 2 and 5 months after casting.

hydrogel, we obtain good interfacial bonds without further processing. Silver-coated conductive thread is used to connect each of the five sensors to a potential divider, which is measured at $10 \mathrm{kHz}$. Time-series resistances from the first two sensors-the thumb and index fingerare plotted, illustrating the clear responses to simple hand gestures, each of which is repeated three times during a $10 \mathrm{~s}$ period (see supplementary video). The rapid prototyping setup enables new sensor morphologies to be quickly proposed, fabricated, and tested and could be used to optimize the sensor response to particular gestures or tasks.

Due to the easy processibility and thermoreversibility of the hydrogel material, it can also be used to mold complex structures. Figure 4c shows a soft pneumatic actuator cast completely with the ionic hydrogel. To the best of our knowledge, this is the first demonstration of a self-healing hydrogel-based pneumatic actuator ${ }^{55}$. As the whole structure is conductive and responsive to strain, the internal state of the actuator can be easily measured by probing any two locations on the actuator. Such devices are appealing for soft robotic applications due to their biocompatibility and recyclability. We also investigate the actuator's self-healing capabilities. Tests are performed on a 60-day-old actuator to mimic real-world scenarios and showcase the stability of the material. Figure $4 \mathrm{c}$ shows the electrical response of the system when the actuator is pierced while undergoing a $0.5 \mathrm{~Hz}$ periodic motion. As the whole actuator is conductive, information about its internal states can be estimated, even after damage. The 
internal pressure recorded in the pressure regulator is also shown here. We perform two healing experiments: one at room temperature $\left(20^{\circ} \mathrm{C}\right)$ and another using a heating device (see supplementary video). At room temperature, the 2-month-old actuator appears to self-heal after just $1 \mathrm{~h}$. However, upon continuous pressurization, it fails to maintain a tight seal. Figure 4c shows how the internal pressures inside the actuator are maintained to an undamaged standard using room temperature healing. A more robust seal can be obtained by heating the hydrogel at the location of damage, although this slightly modifies the actuator's physical properties. Figure $4 \mathrm{c}$ also shows that the actuator does not degrade over a 5 month period.

\section{Conclusions}

In this work, we demonstrate the impressive electrical and mechanical properties of an ionically sensorized selfhealing gelatin hydrogel. The material can be cast or 3D printed using a low-cost setup to create customizable sensor networks and sensorized actuators that have highly linear responses to strain. Table 2 summarizes a number of the key characteristics of the $\mathrm{Ge} / \mathrm{Gl} / \mathrm{H}_{2} \mathrm{O} / \mathrm{CA} / \mathrm{NaCl}$ hydrogel, many of which surpass those of existing sensorized hydrogels and silicones used in soft robotics. Notably, strains exceeding $450 \%$ are achieved, with a highly linear strain response measured up to $e=300 \%$. A reasonable gauge factor is demonstrated, and strain sensitivity can be recovered at room temperature within an hour of damage. Our hydrogel is preferable to many existing alternatives due to its long-term stability without drying out and its fabrication entirely from widely available, food-safe materials. Additionally, its thermoreversibility and low sol-gel transition temperature make it a promising candidate for the straightforward printing of custom strain sensors, as well as providing a method of thermal healing to reliably recover strains of $250 \%$. The authors' previous work ${ }^{45}$ used carbon conductive paint to sensorize chemically crosslinked gelatin hydrogels with GF $\approx 2.5$, which quickly dried out and minimally reduced the baseline resistance. Another self-healing gelatin hydrogel using tannic acid ${ }^{30}$ achieves considerably higher strains of $\sim 1500 \%$ but requires silver nanowires for its sensorization to a lower gauge factor. We have demonstrated extremely linear sensor responses with $\mathrm{GF}=1.49$ up to $300 \%$ using only salt as a sensorization additive, making our material a good choice for biocompatible and low-cost sensing within this range.

The material properties and sensitivities of the proposed hydrogel do not degrade over long periods of time, and the printed samples stabilize to laboratory environmental conditions after losing only $20 \%$ of their mass. Large increases in conductivity are possible using small ratios of $\mathrm{NaCl}$, producing stress-strain responses similar to that of the pure hydrogel and minimizing interfacial stresses in integrated systems. Furthermore, when damaged shortly after printing, the material is capable of self-healing to reach $>100 \%$ strain at room temperature. Extending this strong healing region beyond the initial desorption period would benefit integration into soft robotic systems: preliminary investigations suggest that rehydration is a promising method but requires further investigation. Healing can be manually achieved through external heating due to the hydrogel's thermoreversibility and low sol-gel transition temperature.

The material's lack of degradation over multiple months in the laboratory makes it a strong candidate for rapid prototyping in this field. Further work should consider the range of conditions under which this stability occurs: $\mathrm{pH}$, temperature, and humidity should be adjusted over longer periods of time. Due to its low resistivity and AC measurements, the energy consumption of our hydrogel sensors is very low, making them appealing for wearable applications.

Hydrogel-based sensors have a strong dependency on the environmental conditions and tend to deteriorate over time. We demonstrate that by using multiple sensors, these environmental factors can be estimated and compensated. Due to their high processability and adhesive properties, these materials can be easily integrated into existing wearable devices and soft robotic applications. The ability to respond to changes in the environment, alongside the custom fabrication method presented for the self-healing sensorized hydrogel, paves the way for further exploration of autonomous robots in medical, edible, wearable, hostile, and agricultural applications.

\section{Materials and methods \\ Conductive hydrogel preparation}

A 1:1.5:2.5:0.2 mass ratio of gelatin (Ge), glycerol (Gl), water $\left(\mathrm{H}_{2} \mathrm{O}\right)$, and citric acid monohydrate $(\mathrm{CA})$ is used for the "pure" mixture. Cold water is poured onto 240/260 pork gelatin powder (Cake SOS) and allowed to bloom for 10 min. Next, glycerol (Fisher Scientific) and citric acid monohydrate (Fisher Scientific) are added, and the beaker is covered with parafilm and transferred to a water bath at $50^{\circ} \mathrm{C}$. Once the mixture is homogeneous, the bath is used to maintain the sol state: in practice, this is left overnight, and experiments are performed the following morning. The sol-gel transition occurs at $\sim 45^{\circ} \mathrm{C}$.

To reduce the hydrogel's baseline resistance while retaining its strain responsiveness, four conductive additives are compared: $100 \mu \mathrm{m}$ milled carbon fiber (CF), ThermoFisher general purpose graphite powder (GP), Liquiwire carbon conductive paint (CCP), and Sainsbury's table salt $(\mathrm{NaCl})$. Unless stated otherwise, all are added to the hydrogel with a 1:0.02 pure:additive mass ratio, stirred, and allowed to reach homogeneity in a water bath at $50^{\circ} \mathrm{C}$ before fabrication. The sensorized mixtures are cast 
into $18 \times 40 \mathrm{~mm}$ polylactic acid (PLA) molds joined to a glass plate using hot melt adhesive. After selecting $\mathrm{NaCl}$ is selected as the sensorization additive, the subsequent experiments use $18 \times 45 \times 1 \mathrm{~mm}$ samples printed using a custom 3D printing setup.

\section{D printer setup}

The 3D printing setup consists of a modified Creality CR-20 fused deposition modeling (FDM) printer (Fig. 2) using two $50 \mathrm{ml}$ syringes as the basis of a dual-nozzle extrusion system. Silicone heating pads are used alongside 2 Pt2000 thermistors to create an Arduino-controlled bang-bang trigger mechanism that retains the hydrogel inside the syringes at $45^{\circ} \mathrm{C}$. The gelatin mixtures can be individually extruded from the $1 \mathrm{~mm}$ nozzle using stepper motors that control the rack-and-pinion syringe depression.

By operating at $45^{\circ} \mathrm{C}$, adjacent lines printed using a high extrusion rate are able to coalesce before solidifying, while slowly extruded thin lines can produce more detailed shapes/networks. This hybrid approach enables the printing of 2D layers that are both detailed and isotropic: the shape's thin boundaries are first printed and then used to contain a coalescing infill. These parameters are controlled using Cura to generate a gcode file that is edited with a custom script before being sent serially to the printer. The dual-nozzle setup enables seamless fabrication of composite structures; Fig. 2 demonstrates the printer's ability to combine sensorized and insulating hydrogel layers to create flexible, stretchable, and biodegradable strain sensors.

\section{Mechanical/Electrical characterization}

Unless stated otherwise, the mechanical properties of the salted samples are characterized using an Instron 5544 extending at $10 \mathrm{~mm} / \mathrm{min}$. Stress and strain values are calculated based on the unstressed cross-sections and exposed lengths. Subsequent strain tests, in which the hydrogel's resistive response is simultaneously measured, are performed by clamping the samples between the table and the end-effector of a Universal Robots UR5 robotic arm. Long-term resistances (during tests of environmental dependence) are measured using direct current (DC) voltages. The samples form one branch of a potential divider, which is probed using an Arduino Duemilanove microcontroller and logged using a serially connected Raspberry Pi 3B. During this logging, environmental conditions are simultaneously tracked using a DHT11 temperature and humidity sensor. To estimate the environmental variables from the raw sensors and to predict the passive response of the sensor, a single-layer feedforward neural network is used. The MATLAB neural network toolbox is used for creating and training the network. The trainlm algorithm with a training, test and validation ratio of 70:15:15 is used to train the network. For the first network, the inputs are the three sensor data, and the output is the two environmental variables: relative humidity and temperature. For the second network, the inputs are the raw values from the first two sensors, and the output is the response of the third sensor.

For shorter-term sensor responses, $\mathrm{NaCl} \mathrm{DC}$ measurements undergo a short period of increasing resistance before stabilizing. This effect, due to the motion of the additional ions through the hydrogel, is eliminated when an AC signal is used due to the repeated reversal of probe polarity. AC measurements $(1 \mathrm{kHz})$ are recorded using a handheld LCR meter (Keysight U1731C), while 10 and $100 \mathrm{kHz}$ logging is performed using a National Instruments USB-6212 multifunction I/O device coupled with a potential divider and sampling at $100 \mathrm{kHz}$. The input/ output voltage ratio is calculated using the relative magnitude of the applied frequency Fourier peaks over a 1000sample rolling window. Capacitive effects at these high frequencies are not explicitly taken into consideration, although the measured resistance of a short circuit is subtracted from all readings. All the data processing is performed using MATLAB.

While comparing the relative salt content, pressure responses are recorded when a $100 \mathrm{~g}$ circular mass of $19 \mathrm{~mm}$ diameter is placed on the printed sample; samples under flexure are bent around a PLA jig with $12 \mathrm{~mm}$ curvature radius; and strain measurements are taken during $25 \mathrm{~mm}$ applied extension. All forces are applied through a thin sheet of insulating polydimethylsiloxane (PDMS) to avoid unintended changes in resistance, and values are given $10 \mathrm{~s}$ to stabilize before being recorded.

The healing tests shown in Fig. $3 \mathrm{c}$ are performed by cutting each sample across its width perpendicular to the axis of strain and leaving the two halves in contact for $48 \mathrm{~h}$ at room temperature before strain testing with the robotic arm setup described above. "Rehydrated" samples are coated on both sides with an excess of warm water before this $48 \mathrm{~h}$ period, while both sides of the thermally healed samples are quickly passed over with a rework station's hot air gun.

The $\mathrm{pH}$ dependence is investigated using a $0.1 \mathrm{M}$ Tris$\mathrm{HCl}$ buffer solution at one of $6 \mathrm{pH}$ values, namely, 6.8, 7.0, $7.5,8.0,8.5$, and 8.8, each of which is tested twice. Then, $0.3 \mathrm{~g}$ of solution is pipetted onto $1 / 50 \mathrm{NaCl}$ samples after solidification in their casts and left for $24 \mathrm{~h}$ before being tested.

Pneumatic actuations of a PneuNet finger are controlled by a Festo $V E A B$ proportional pressure regulator, the input signal to which is provided by a National Instruments USB-6212 multifunction I/O device. This I/O device also provides a $5 \mathrm{kHz}$ signal for resistive measurements. The actuator is cast using the low water composition from a 2-part PLA mold and sealed using localized heating. The base is sensorized using $1 / 50 \mathrm{NaCl}$, 
and conductive thread is joined to its ends using the sol hydrogel. Damage is inflicted by piercing a scalpel through the finger's underside into one of the inflatable chambers. A rework station's hot air gun is used for localized healing of the actuator.

\section{Acknowledgements}

This work was supported by the SHERO project, a Future and Emerging Technologies (FET) program of the European Commission (grant agreement ID 828818) and by EPSRC DTP EP/R513180/1. The authors would like to thank Alex Casabuena Rodriguez and Professor Michael Sutcliffe for their assistance in characterizing the hydrogel's stress-strain response and George Wood for the preparation of the Tris-HCl buffer solutions.

\section{Author contributions}

All authors conceived the work and reviewed the results. D.H. \& T.G.T. conducted the experiments and wrote the manuscript.

\section{Competing interests}

The authors declare no competing interests.

\section{Publisher's note}

Springer Nature remains neutral with regard to jurisdictional claims in published maps and institutional affiliations.

Supplementary information The online version contains supplementary material available at https://doi.org/10.1038/s41427-022-00357-9.

Received: 1 October 2021 Revised: 17 December 2021 Accepted: 11 January 2022.

Published online: 18 February 2022

\section{References}

1. Amjadi, M., Kyung, K-U., Park, I. \& Sitti, M. Stretchable, skin-mountable, and wearable strain sensors and their potential applications: a review. Adv. Funct. Mater. 26, 1678-1698 (2016).

2. Rus, D. \& Tolley, M. T. Design, fabrication and control of soft robots. Nature 521, 467-475 (2015).

3. Mattmann, C., Clemens, F. \& Tröster, G. Sensor for measuring strain in textile. Sensors 8, 3719-3732 (2008).

4. Georgopoulou, A. \& Clemens, F. Piezoresistive elastomer-based composite strain sensors and their applications. ACS Appl. Electron. Mater. 2, 1826-1842 (2020).

5. Sareh, S. et al. Bio-inspired tactile sensor sleeve for surgical soft manipulators. In 2014 IEEE International Conference on Robotics and Automation (ICRA) 1454-1459 (IEEE, 2014).

6. Culha, U., Nurzaman, S. G., Clemens, F. \& lida, F. Svas3: strain vector aided sensorization of soft structures. Sensors 14, 12748-12770 (2014).

7. Kramer, R. K., Majidi, C., Sahai, R. \& Wood, R. J. Soft curvature sensors for joint angle proprioception. In 2011 IEEE/RSJ International Conference on Intelligent Robots and Systems, 1919-1926 (IEEE, 2011).

8. Russo, S. et al. Soft and stretchable sensor using biocompatible electrodes and liquid for medical applications. Soft Robot. 2, 146-154 (2015).

9. Yang, C. \& Suo, Z. Hydrogel ionotronics. Nat. Rev. Mater. 3, 125-142 (2018).

10. Lee, Y., Song, W. J. \& Sun, J.-Y. Hydrogel soft robotics. Mater. Today Phys. 15, $100258(2020)$

11. Cai, G. et al. Extremely stretchable strain sensors based on conductive selfhealing dynamic cross-links hydrogels for human-motion detection. Adv. Sci. 4, 1600190 (2017).

12. Cheng, S., Narang, Y. S., Yang, C., Suo, Z. \& Howe, R. D. Stick-on large-strain sensors for soft robots. Adv. Mater. Interfaces 6, 1900985 (2019).

13. Tong, R. et al. Highly stretchable and compressible cellulose ionic hydrogels for flexible strain sensors. Biomacromolecules 20, 2096-2104 (2019).

14. Han, L. et al. Mussel-inspired adhesive and conductive hydrogel with longlasting moisture and extreme temperature tolerance. Adv. Funct. Mater. $\mathbf{2 8}$ 1704195 (2018).
15. He, P. et al. Anti-freezing and moisturizing conductive hydrogels for strain sensing and moist-electric generation applications. J. Mater. Chem. A 8, 3109-3118 (2020).

16. Shao, Z. et al. Degradable self-adhesive epidermal sensors prepared from conductive nanocomposite hydrogel. Nanoscale 12, 18771-18781 (2020).

17. Huang, J. et al. A dual-mode wearable sensor based on bacterial cellulose reinforced hydrogels for highly sensitive strain/pressure sensing. Adv. Electron. Mater. 6, 1900934 (2020).

18. Hong, W., Zhao, X., Zhou, J. \& Suo, Z. A theory of coupled diffusion and large deformation in polymeric gels. J. Mech. Phys. Solids 56, 1779-1793 (2008).

19. Yuk, H. et al. Hydraulic hydrogel actuators and robots optically and sonically camouflaged in water. Nat. Commun. https://doi.org/10.1038/ncomms 14230 (2017).

20. Peng, X. \& Wang, H. Shape changing hydrogels and their applications as soft actuators. J. Polym. Sci. Part B 56, 1314-1324 (2018).

21. Bennett, D. NaCl doping and the conductivity of agar phantoms. Mater. Sci. Eng. C31, 494-498 (2011).

22. Keplinger, C. et al. Stretchable, transparent, ionic conductors. Science 341, 984-987 (2013).

23. Liu, S. \& Li, L. Ultrastretchable and self-healing double-network hydrogel for 3D printing and strain sensor. ACS Appl. Mater. Interfaces 9, 26429-26437 (2017).

24. Tian, $\mathrm{K}$. et al. 3D printing of transparent and conductive heterogeneous hydrogel-elastomer systems. Adv. Mater. 29, 1604827 (2017).

25. Pan, X. et al. Ultraflexible self-healing guar gum-glycerol hydrogel with injectable, antifreeze, and strain-sensitive properties. ACS Biomater. Sci. Eng. 4, 3397-3404 (2018)

26. Sun, W., Schaffer, S., Dai, K., Yao, L. \& Feinberg, A. 3D printing hydrogel-based soft and biohybrid actuators: a mini-review on fabrication techniques, applications, and challenges. Front. Robot. Al 8, 1-10 (2021).

27. Ouyang, L., Yao, R., Zhao, Y. \& Sun, W. Effect of bioink properties on printability and cell viability for 3D bioplotting of embryonic stem cells. Biofabrication $\mathbf{8}$, 035020 (2016).

28. Stevenson, M. et al. Development and characterization of ribose-crosslinked gelatin products prepared by indirect 3D printing. Food Hydrocoll. 96, 65-71 (2019).

29. Sardesai, A. N. et al. Design and characterization of edible soft robotic candy actuators. MRS Adv. 3, 3003-3009 (2018).

30. Wang, J. et al. Self-healing and highly stretchable gelatin hydrogel for selfpowered Strain sensor. ACS Appl. Mater. Interfaces 12, 1558-1566 (2020).

31. Yan, X. et al. High strength and self-healable gelatin/polyacrylamide double network hydrogels. J. Mater. Chem. B 5, 7683-7691 (2017).

32. Zhang, G., LV, L., Deng, Y. \& Wang, C. Self-healing gelatin hydrogels crosslinked by combining multiple hydrogen bonding and ionic coordination. Macromol. Rapid Commun. 38, 1-9 (2017).

33. Sanwlani, S., Kumar, P. \& Bohidar, H. B. Hydration of gelatin molecules in glycerol-water solvent and phase diagram of gelatin organogels. J. Phys. Chem. B 115, 7332-7340 (2011).

34. Bigi, A., Panzavolta, S. \& Rubini, K. Relationship between triple-helix content and mechanical properties of gelatin films. Biomaterials 25, 5675-5680 (2004).

35. Peppas, N. A., Hilt, J. Z., Khademhosseini, A. \& Langer, R. Hydrogels in biology and medicine: from molecular principles to bionanotechnology. Adv. Mater. 18, 1345-1360 (2006)

36. Lukasik, K. V. \& Ludescher, R. D. Molecular mobility in water and glycero plasticized cold- and hot-cast gelatin films. Food Hydrocoll. 20, 96-105 (2006).

37. Invine, S. A. et al. Printing cell-laden gelatin constructs by freeform fabrication and enzymatic protein crosslinking. Biomed. Microdevices 17, 1-8 (2015).

38. Tan, J. J. Y., Lee, C. P. \& Hashimoto, M. Preheating of gelatin improves its printability with transglutaminase in direct ink writing 3D printing. Int. J. Bioprinting 6, 118-129 (2020).

39. Shintake, J., Sonar, H., Piskarev, E., Paik, J. \& Floreano, D. Soft Pneumatic Gelatin Actuator for Edible Robotics 6221-6226 (IEEE, 2017).

40. Han, L. et al. Mussel-inspired adhesive and conductive hydrogel with longlasting moisture and extreme temperature tolerance. Adv. Funct. Mater. 28, 1-12 (2018).

41. Bergo, P. \& Sobral, P. J. Effects of plasticizer on physical properties of pigskin gelatin films. Food Hydrocoll. 21, 1285-1289 (2007).

42. Piazzoni, M. et al. Monolithic three-dimensional functionally graded hydrogels for bioinspired soft robots fabrication. Soft Robot. 00, 1-9 (2021).

43. Nagai, T., Kurita, A. \& Shintake, J. Characterization of sustainable robotic materials and finite element analysis of soft actuators under biodegradation. Front. Robot. A/ 8, 383 (2021). 
44. Hughes, J. \& Rus, D. Mechanically programmable, degradable ingestible soft actuators. 2020 3rd IEEE International Conference on Soft Robotics, RoboSoft 2020 836-843, https://doi.org/10.1109/RoboSoft48309.2020.9116001 (2020).

45. Hardman, D., Hughes, J., Thuruthel, T. G., Gilday, K. \& lida, F. 3D printable sensorized soft gelatin hydrogel for multi-material soft structures. IEEE Robot. Autom. Lett 6, 5269-5275 (2021).

46. Baumgartner, M. et al. Resilient yet entirely degradable gelatin-based biogels for soft robots and electronics. Nat. Mater. 19, 1102-1109 (2020).

47. Thuruthel, T. G., Hughes, J., Georgopoulou, A., Clemens, F. \& lida, F. Using redundant and disjoint time-variant soft robotic sensors for accurate static state estimation. IEEE Robot. Autom. Lett. 6, 2099-2105 (2021).

48. Liu, Y. \& Hsu, S. H. Synthesis and biomedical applications of self-healing hydrogels. Front. Chem. 6, 1-10 (2018).

49. Thangavel, G., Tan, M. W. M. \& Lee, P. S. Advances in self-healing supramolecular soft materials and nanocomposites. Nano Converg. https:/doi.org/ 10.1186/s40580-019-0199-9 (2019).
50. Phadke, A. et al. Rapid self-healing hydrogels. Proc. Natl Acad. Sci. USA 109 4383-4388 (2012)

51. Xu, Y., Yang, H., Zhu, H., Jiang, L. \& Yang, H. Self-healing gelatin-based shape memory hydrogels via quadruple hydrogen bonding and coordination crosslinking for controlled delivery of 5-fluorouracil. J. Biomater. Sci. Polym. Ed. 31, 712-728 (2020).

52. Wang, J. et al. Self-healing and highly stretchable gelatin hydrogel for self-powered strain sensor. ACS Appl. Mater. Interfaces 12, 1558-1566 (2019).

53. Li, C. H. \& Zuo, J. L. Self-healing polymers based on coordination bonds. Adv Mater. https://doi.org/10.1002/adma.201903762 (2020).

54. Mao, J., Kondu, S., Ji, H. F. \& McShane, M. J. Study of the near-neutral pHsensitivity of chitosan/gelatin hydrogels by turbidimetry and microcantilever deflection. Biotechnol. Bioeng. 95, 333-341 (2006).

55. Terryn, S. et al. A review on self-healing polymers for soft robotics. Mater. Today 47, 187-205 (2021). 